Recherches en didactique des langues et des cultures

Les cahiers de l'Acedle

7-1 | 2010

Notions en questions - Les plurilinguismes

\title{
Diversité des plurilinguismes et formes de l'éducation plurilingue et interculturelle
}

Daniel Coste

\section{(2) OpenEdition}

1 Journals

Édition électronique

URL : https://journals.openedition.org/rdlc/2031

DOI : $10.4000 /$ rdlc.2031

ISSN : 1958-5772

Éditeur

ACEDLE

Référence électronique

Daniel Coste, «Diversité des plurilinguismes et formes de l'éducation plurilingue et interculturelle», Recherches en didactique des langues et des cultures [En ligne], 7-1 | 2010, mis en ligne le 01 avril 2010, consulté le 12 avril 2022. URL : http://journals.openedition.org/rdlc/2031 ; DOI : https://doi.org/ $10.4000 /$ rdlc. 2031

Ce document a été généré automatiquement le 12 avril 2022.

\section{(1) () (9)}

Recherches en didactique des langues et des cultures is licensed under a Creative Commons AttributionNonCommercial-NoDerivatives 4.0 International License 


\title{
Diversité des plurilinguismes et formes de l'éducation plurilingue et interculturelle
}

\author{
Daniel Coste
}

\section{Introduction}

1 Pour cette journée de la série Notions en question(s), le propos est de répondre ici, au moins en partie, à l'interrogation initiale posée par les organisateurs : "Les approches didactiques "plurielles" se réfèrent-elles à une forme "universelle" de plurilinguisme ou prennent-elles en compte ces divers "plurilinguismes" ? Comment? Avec quels objectifs ?". Mais l'intitulé même de la journée ("Diversité et hétérogénéité des plurilinguismes") constitue une prise de position et invite aussi à questionner certaines lectures unifiantes ou par trop génériques de la notion même de plurilinguisme.

2 Les variations contextuelles et les variations individuelles du plurilinguisme conduisent-elles à considérer qu'il conviendrait, tant pour des raisons d'ordre épistémique qu'en regard des options didactiques, de sous-catégoriser différentes formes ou types de plurilinguisme, s'agissant aussi bien des pratiques que des représentations?

3 Mon expérience m'a rendu plus familier des contextes européens que d'autres "terrains" et l'apport de Bruno Maurer, touchant plus ces derniers, est susceptible de compléter ou contester mes propres commentaires. Mais l'Europe elle-même ne manque ni de diversité ni d'hétérogénéité... ${ }^{1}$

4 Dans notre domaine comme dans d'autres aujourd'hui, l'heure est à prêter attention à la pluralité, à la variabilité, à la différenciation. Peut-être parce que, par ailleurs, les forces tendant à la standardisation et à l'uniformité sont perçues, à tort ou à raison, comme particulièrement actives. Rien d'étonnant donc, à ce que tout ce qui semblerait favoriser une doxa uniformisante soit soumis à examen, y compris - non sans quelque paradoxe apparent - lorsqu'il s'agit de notions en vogue comme celle de plurilinguisme. 
Ce qui suit s'ordonne en deux moments principaux :

- un essai d'exploration de quelques critères qui permettraient de définir et de catégoriser une pluralité de plurilinguismes ;

- une reconnaissance des perspectives ouvertes par une éducation plurilingue et interculturelle qui tout à la fois prendrait la pleine mesure de cette affirmation d'une différenciation des plurilinguismes et se donnerait des repères et des principes d'action qui ne soient pas uniquement contextuels et à valeur locale.

Entre ces deux parties, deux développements distincts mais voulus complémentaires :

- un questionnement portant sur les Profils de politique linguistique éducative proposés aux États-membres par le Conseil de l'Europe ;

- une relecture rapide de l'évolution de la notion de compétence plurilingue.

\section{Des plurilinguismes?}

\subsection{Petits frottements (pas seulement) terminologiques}

7 La notion de plurilinguisme semble désormais bien établie en sociolinguistique et en didactique des langues, avec la distinction elle aussi devenue courante entre plurilinguisme des individus et multilinguisme des territoires, l'un n'impliquant pas l'autre et inversement ${ }^{2}$. A condition toutefois de s'en tenir au français, car la distinction ainsi posée n'est pas toujours reproduite dans le lexique d'autres langues. Et, surtout, dans le cas de l'anglais, multilingualism tend à occuper tout l'espace et à plus ou moins recouvrir les deux dimensions (individuelle et territoriale). Le Conseil de l'Europe adopte certes pour les textes en français et en anglais le partage multilinguismemultilingualism / plurilinguisme-plurilingualism et promeut le plurilinguisme comme "manière d'être en Europe" (Beacco, 2005), mais la Commission européenne et son Commissariat au multilinguisme s'en tiennent, y compris pour le français, au seul multilinguisme / multilingualism. ${ }^{3}$

8 Faut-il noter en outre que, dans nombre de pays européens, pour ce qui est de l'insertion des migrants, les orientations dominantes ne font état que de l'apprentissage de la langue du pays d'accueil ${ }^{4}$ ? On en arriverait ainsi bientôt à estimer que le terme et la notion de "plurilinguisme" sont en effet quasi superflus et que, du coup et a fortiori, s'interroger sur la pluralité des plurilinguismes relève, loin des réalités à venir, de l'exercice gratuit pour journées d'études entre francophones.

En bref, pour en rester aux constats plutôt qu'aux commentaires, mettre en question(s) aujourd'hui la notion de "plurilinguismes" (au pluriel) ne saurait faire oublier que le plurilinguisme des individus n'est ni partout reconnu, ni généralement présenté comme un objectif, ni pleinement considéré comme véritable objet d'étude.

\subsection{Quels critères de catégorisation distinctive des plurilinguismes?}

Pour les tenants d'un plurilinguisme caractérisé comme le développement et l'usage par les acteurs sociaux d'une capacité à disposer des ressources d'expression de plusieurs langues, qu'en est-il ? Le fait et l'argument mis en avant sont que le plurilinguisme des individus est quasiment la chose la mieux partagée du monde, un 
phénomène très répandu, non seulement hors d'Europe mais aussi en Europe ${ }^{5}$. C'est le monolinguisme qui devient l'exception, le plurilinguisme la presque règle.

11 Mais cette reconnaissance du plurilinguisme "ordinaire", vu comme résultant de divers facteurs historiques, régionaux, ethniques, religieux... et bien différent de ce que peut produire l'enseignement scolaire des langues "étrangères", s'accompagne d'une prise de conscience de la multiplicité des cas de figure auxquels il donne lieu. D'où l'intérêt intrinsèque d'une description ordonnée, d'un classement de ces cas de figure comme déterminant des types bien distincts de configurations, voire - on y revient - une pluralité de plurilinguismes. Et les enjeux ne tiennent pas seulement de l'ordre du descriptif : les débats autour du plurilinguisme ordinaire se focalisent sur les avantages et les inconvénients qu'il comporterait, notamment pour la scolarisation des enfants; distinguer divers types de plurilinguisme pourrait aider à nuancer les prises de position, à dépasser des oppositions trop tranchées et à fonder des interventions didactiques mieux ajustées.

Les critères permettant de différencier entre plusieurs (formes de) plurilinguismes sont évidemment multiples et c'est la combinatoire entre tels et tels d'entre eux qui devrait autoriser des catégorisations à l'intérieur d'un ensemble perçu comme hétérogène.

Sans prétendre aboutir à des propositions de catégories, il est relativement aisé d'aligner des séries de spécifications pouvant entrer dans des combinaisons de critères. Celles qui suivent sont (à dessein) de nature diverse. 
- plurilinguisme revendiqué / caché / honteux

- plurilinguisme assuré / insécure (du point de vue formel / statutaire / identitaire)

Quelques remarques s'imposent à propos de cette esquisse empirique sommaire :

- ces séries de distinctions demanderaient à être précisées et illustrées (mais sont sans doute suffisamment évocatrices pour les lecteurs) ;

- elles sont hétérogènes entre elles, les critères pris en considération relevant de différents points de vue sur le plurilinguisme; mais cette hétérogénéité importe, à raison de la diversité des dimensions à l'œuvre ;

- pour la plupart d'entre elles, sans surprise, le plurilinguisme peut apparaître comme un mixte (par exemple : d'origine à la fois familiale et scolaire) ;

- il existe, à l'évidence, des recoupements entre les différents angles de saisie (ainsi, l'exposition scolaire officielle porte le plus souvent sur des langues centrales, dominantes, littéraciées et normées) ;

- le nombre de compositions possibles de ces différents critères est clairement très élevé et toute la question est de déterminer, en relation à un contexte donné, le niveau utile (à des fins de description ou d'intervention) de catégorisation d'un plurilinguisme marqué par tel (sous-)ensemble de traits ;

- une approche contextualisée permet ainsi de mettre en évidence des plurilinguismes, mais dont chacun, bien que puisant à la même base de traits caractérisants, en mobilise une certaine sélection qui ne recouvre pas nécessairement les mêmes traits variables que d'autres.

L'hypothèse ici sous-jacente est que catégoriser des plurilinguismes peut difficilement se faire sur la seule base d'un seul type de critères (d'ordre linguistique ou sociolinguistique ou sociologique ou fonctionnel ou encore acquisitionnel).

Il y aurait aussi lieu d'introduire des considérations d'ordre plus cognitif et processuel et de faire la distinction entre des formes de plurilinguisme qu'on pourrait dire additives / cumulatives où les différentes ressources langagières à disposition semblent demeurer nettement séparées (ce qui n'implique pas nécessairement des cloisonnements dans la mobilisation de ces ressources) et d'autres formes, qu'on pourrait dire (re)compositionnelles, où des sortes d'osmoses, de passages paraissent beaucoup plus à l'œuvre dans la construction et la gestion cognitives des ressources langagières relevant d'origines et de systèmes distincts. Mais je ne m'aventurerai pas plus avant sur ce terrain, important qu'il soit, car il reste, à mes yeux et pour l'heure, beaucoup plus mouvant que d'autres (Castellotti, 2001; Castellotti et Moore, 2005 ; et aussi, d'autre perspective : Cenoz \& Genesee, 1998).

Une chose est certaine : les séries grossières de traits distinctifs alignées plus haut ne sauraient alimenter la catégorisation pour le plaisir de la catégorisation ni réveiller le démon de la taxinomie. Autant il importe de ne pas faire du plurilinguisme une entité homogène dotée partout et pour tous des mêmes caractéristiques, autant prendre en compte tous les facteurs de différenciation possibles pour inventorier une infinité de plurilinguismes aboutirait à une atomisation aussi coûteuse que vaine, tant scientifiquement qu'en termes d'action de politique linguistique ou de didactique.

La difficulté consiste dès lors à concilier deux options méthodologiques: d'un côté partir des contextes et des acteurs et non projeter sur ceux-ci une grille de lecture unique et a priori; de l'autre se garder d'un travail purement monographique et local 
qui ne pourrait se faire, cumulativement, qu'au détriment d'une réflexion et d'une intervention tant soit peu transversales.

Faut-il donc hiérarchiser les différents critères évoqués ou, du moins, poser certains traits comme les plus structurants de la pluralité des plurilinguismes et comme à prendre en considération en priorité ?

Pour ce qui est des contextes européens et des individus qui y vivent et y sont ou y ont été scolarisés, on peut à cet égard souligner l'importance du statut et des caractéristiques linguistiques et sociolinguistiques de la langue première, ainsi que du rapport de celle-ci avec la langue de scolarisation ${ }^{6}$. Il n'y a rien de paradoxal à placer les relations entre répertoire premier de l'individu et langue de scolarisation au centre de toute considération sur les plurilinguismes. On y reviendra.

Mais on conçoit vite, par ailleurs, que des cas - si différents qu'ils soient à nombre d'égards - comme ceux des créoles et des langues régionales, interrogent la notion même de langue dans la mesure où leur description tend à les représenter non comme des systèmes homogènes et unifiés, mais comme constitutivement pluriels et hétérogènes. Bien qu'entrant dans des perspectives et des modélisations très différentes et non limitées aux créoles et langues régionales, des notions comme celles de grammaire polylectale (Berrendonner, Le Guern et Puech, 1983), d'interlecte (Prudent, 2005), de polynomie (Marcellesi, 2003) troublent les concepts de variété et de variation et repositionnent autrement celui de langue (voir Billiez, 2005 ; Blanchet, Calvet \& De Robillard, 2007). Pour le présent propos, le questionnement devient aussi : où commence le plurilinguisme?

\subsection{Points de vue}

Lüdi et Py (2002) ont proposé, s'agissant de la communication exolingue, de distinguer trois types de points de vue :

- celui de la description "objective" recherchant dans le corpus considéré des traces (constituées comme telles) de gestion de l'exolinguisme ${ }^{7}$;

- celui de la participation, les interlocuteurs définissant et traitant ou non leur interaction comme de nature exolingue ;

- celui de l'interprétation d'un chercheur qui mènerait son travail en posant que toute communication est exolingue, dans la mesure où les interactants possèdent toujours - tant soit peu - des répertoires distincts.

On conviendra que, dans la plupart des travaux de didactique des langues ainsi que dans nombre de ceux relatifs à l'acquisition, la distinction natif / non natif y ayant massivement et durablement cours, la langue du natif, constituée en norme, est de facto postulée une et homogène et que, plus généralement, les deux premiers points de vue nommés ci-dessus prévalent largement sur le troisième.

Doit-on, pour le plurilinguisme, par analogie et extension de la distinction suggérée par Lüdi et Py et en prenant en compte la question formulée ci-dessus : "où commence le plurilinguisme", envisager les trois points de vue: observation/description des pratiques, participation des acteurs, interprétation selon une option posant que tout acteur social est un plurilingue en devenir, quand même il ou elle ne "connaît" qu'une seule "langue", pour autant que celle-ci soit conçue comme polylectale? 
Et, à retenir le troisième de ces points de vue, à considérer que toute communication, tout apprentissage et tout enseignement mettent en contact des répertoires distincts et relèvent donc d'ajustements circonstanciels, voire d'altérations plus durables de ressources langagières ainsi placées en interaction (Coste, 2009), on postule, sinon une cohérence et une continuité totales, du moins une intégration de l'ensemble du répertoire, pour hétérogène qu'il soit, sous le mode d'une gestion de la pluralité et non en termes de cloisonnement et de sélection entre langues ou variétés juxtaposées. Postulat qui consiste aussi à renverser les termes et les enjeux: ne pas penser le plurilinguisme comme une extension / complexification du bilinguisme, lui-même considéré comme résultant d'une adjonction à la langue première, mais bien voir le "bi" et le "mono" comme des cas particuliers du "pluri". S'en tenir ici aux préfixes esquive évidemment la question déjà plus qu'effleurée de la désignation catégorielle de ce qui suit... mais n'entre peut-être pas en contradiction avec le projet de distinguer des (formes de) plurilinguismes, ainsi qu'on l'a amorcé plus-haut. Disons que la mise de notions en question(s) autorise, pour un jour, ce genre de dérive un peu provocatrice ${ }^{8}$.

\section{Retour sur la notion de compétence plurilingue et parcours de politique linguistique}

\subsection{De la diversification du même à l'intégration de l'autre}

Quand la notion de compétence plurilingue est mise sur le marché des politiques linguistiques et de la didactique des langues (Coste, Moore, Zarate, 1997) elle intéresse d'abord l'enseignement des langues étrangères. Définie comme à la fois une et plurielle, elle se présente comme capacité à gérer et à étendre un répertoire de langues et variétés maîtrisées à des degrés divers. Il s'agit de sortir de représentations et de pratiques reposant sur les images mythiques du natif (Renaud, 1998) et du "parfait bilingue" et sur des modèles de l'apprentissage de plusieurs langues comme d'un cumul de compétences à la fois homologues et bien séparées.

La complexification s'opère du moment que sont prises en compte non seulement les langues étrangères reconnues et enseignées par l'école, mais aussi les autres éléments de leur répertoire que les apprenants doivent à leur famille, à leur environnement, à leurs activités avant et à côté de la scolarisation. Les composantes (langues, variétés, pratiques langagières de communautés) entrant dans le champ de ce que mobilise la compétence plurilingue paraissent ainsi plus diverses à tous égards, quant à leurs rôles communicationnels respectifs, leur degré de reconnaissance sociale, les valeurs affectives et cognitives qui s'y attachent, leurs places variables dans les jeux et enjeux identitaires et les appartenances multiples de l'acteur social considéré (Coste, 2004 ; Moore, 2006, Moore \& Castellotti, 2008; Stratilaki, 2008; Castellotti et al., 2009, ). Où l'on retrouve, sous un autre angle mais sans surprise, les dimensions hétérogènes qui servaient tout à l'heure à lister des critères possibles dont la composition serait susceptible de catégoriser des plurilinguismes.

Caractérisée comme la capacité à mobiliser et à faire évoluer les ressources langagières d'un répertoire (hétérogène) en vue de leur mise en œuvre dans des familles de situations à des fins de résolution de tâches et d'interaction sociale, la compétence plurilingue est représentée comme activant des dispositions et des attitudes, des savoir apprendre, des savoirs et savoir faire plus ou moins accessibles, plus ou moins 
combinables, l'hétérogénéité du répertoire tenant aussi à ce que certaines composantes en sont fluides, osmotiques, alors que d'autres peuvent s'avérer figées ou fossilisées. Les rapports entre les pratiques et représentations de l'individu et celles des diverses communautés dans lesquelles il s'inscrit et intervient ont évidemment une part dans ces décalages internes de mobilisation et d'évolution des composantes du répertoire, autant que (et en relation avec) les dimensions linguistiques et cognitives (Lüdi, 2004) ${ }^{9}$.

Dans le travail accompli sur la notion de compétence plurilingue, le passage d'une conception encore additive de la diversification des langues enseignées à un accent mis sur le répertoire plurilingue préexistant et/ou parallèle de nombre des apprenants, puis à la prise en compte d'un déjà là langagier pluriel pour tous, la langue première et la langue majeure de scolarisation (qui ne se confondent pas), voire une ou d'autres langues de référence, sont mises en évidence, non seulement comme parties intégrantes de toute compétence plurilingue, mais comme y tenant des rôles déterminants pour l'équilibre et le développement de l'ensemble. Nouveau recoupement avec les interrogations sur les départs possibles entre différents plurilinguismes, telles qu'elles ont été esquissées en 1.2.

\subsection{Plurilinguismes et Profils de politique linguistique éducative}

L'importance des langues premières et des langues de scolarisation dans la constitution des compétences plurilingues et dans la différenciation en contexte de plusieurs plurilinguismes peut être illustrée par les données que fournit une des actions que la Division des politiques linguistiques du Conseil de l'Europe propose aux pays membres : l'aide à l'établissement de profils de politique linguistique éducative, à savoir une assistance en vue d'une analyse de la situation sociolinguistique multilingue du pays considéré et de son environnement, ainsi que des choix éducatifs quant aux langues; le tout devant contribuer à d'éventuelles évolutions dans la politique linguistique éducative (Beacco \& Byram, 2003-2007) ${ }^{10}$.

A partir en particulier des Profils établis pour l'Estonie, l'Irlande, la Lituanie, la Norvège, la République slovaque, que je connais mieux que d'autres pour y avoir été directement impliqué, plusieurs constats se dégagent.

Dans tous les cas, la demande initiale du pays touche au développement qualitatif et quantitatif des capacités plurilingues des citoyens et tout particulièrement des élèves scolarisés.

Dans quasiment tous les cas, sauf l'Estonie (encore en cours), cette demande initiale concernait les langues étrangères enseignées ${ }^{11}$. Seule l'Irlande soulignait aussi le besoin d'une stratégie d'ensemble.

41 Dans tous les cas, des circonstances de prime abord "particulières" affectent la ou les langue(s) de scolarisation ${ }^{12}$.

42 - Dans tous les cas, le statut de la langue de scolarisation a à voir, ne serait-ce qu'historiquement, avec d'autres langues présentes dans le pays :

- clair pour l'Irlande dans la relation entre irlandais et anglais ;

- évident pour l'Estonie, la Lituanie, la Slovaquie dans le rapport au russe et/ou au polonais ou au hongrois ${ }^{13}$ : 
- autre mais clair aussi pour la Norvège où la coexistence bokmål/nynorsk est le produit d'un long processus ayant abouti à une sorte de compromis historique, y compris dans la mise à distance du danois.

43 Ces situations pourraient en effet toutes paraître singulières, mais je crois qu'il faut plutôt, méthodologiquement, les voir comme relevant de cet "effet de loupe" révélateur de fonctionnements et relations de portée beaucoup plus générale, à savoir, pour notre propos et de constat trivial, qu'il y a toujours interdépendance, dans une "niche écologique" donnée, entre l'ensemble des langues qui s'y trouvent, d'une manière ou d'une autre, en contact.

On peut ainsi poser :

- que, quel que soit le contexte, la langue de scolarisation ne peut être considérée en ellemême et pour elle-même, indépendamment de celles qui coexistent avec elle; écologiquement, elle a à voir avec son environnement multilingue et les évolutions de celui$\mathrm{Ci}^{14}$;

- que donc, dans les choix de politique linguistique valorisant le plurilinguisme, la situation de la langue de scolarisation ne peut être posée comme complètement distincte et en quelque sorte réglée ;

- que des effets de voisinage peuvent s'observer : ainsi, en Lituanie, la volonté de restauration, de consolidation, de développement et de normalisation du lituanien a eu pour corollaire, à de hauts niveaux de responsabilité, des résistances à l'enseignement d'une langue étrangère aux jeunes enfants du primaire et une méfiance à l'encontre de formes d'enseignement bilingue, CLIL ou EMILE ${ }^{15}$;

- qu'une même langue n'est pas la même dans des contextes différents : la position du russe n'est pas en Estonie celle qu'il a en Lituanie, et cela non simplement pour des raisons de pourcentage de la population et d'évolution démographique, mais aussi parce qu'en Lituanie les questions touchant à la minorité polonophone sont plus vives et plus visibilisées que celles touchant à la minorité russophone ${ }^{16}$;

- que la question des rapports entre langue de scolarisation comme matière et langue de scolarisation dans les autres matières est à la fois omniprésente et très souvent peu conscientisée, peu conceptualisée, voire passée sous silence, ou encore soulevée sous l'angle des choix de politique linguistique plus qu'en termes éducatifs et de gestion de l'apprentissage.

Dans tous ces contextes, diverses formes de plurilinguisme sont observables et leur développement individuel, statistiquement au moins, sont difficilement séparables de ce que sont les situations multilingues, historiquement, régionalement et souvent socio-économiquement marquées des pays considérés ${ }^{17}$. On retrouve aussi sans surprise le constat selon lequel les populations minoritaires sont plus "contraintes" au plurilinguisme que les locuteurs de la langue majoritaire officielle : les hungarophones de Slovaquie sont tenus d'apprendre le slovaque, alors que le hongrois n'est quasiment pas offert aux élèves de la majorité slovaque ${ }^{18}$.

Faisons le point après ces apparents excursus. Qu'il s'agisse des évolutions de la notion de compétence plurilingue ou de la manière dont on peut en analyser certaines manifestations à l'occasion de l'établissement de profils de politique linguistique éducative, les critères possiblement classificatoires rapidement inventoriés supra refont vite surface et permettent de différencier par leur combinatoire une multiplicité des formes (ou formats ?) de plurilinguismes. Mais se trouve aussi renforcée l'hypothèse 
que, dans les constructions et usages divers de ces différents plurilinguismes, trois ordres de facteurs affectent particulièrement le plurilingue en devenir :

- le statut et la reconnaissance de ses pratiques langagières premières, antérieures et parallèles à la scolarisation ;

- leur mise en relation avec la (ou les) langue(s) de scolarisation et le type de traitement scolaire de celle(s)-ci ;

- les (dés)équilibres, tensions et évolutions qui marquent en termes économiques, politiques, symboliques et identitaires l'environnement multilingue dans lequel s'inscrit son développement propre d'acteur social.

Selon les contextes, les déterminismes sociaux pèsent plus ou moins lourd sur les individus et les groupes quant aux marges d'initiative dont ils disposent pour gérer au mieux leur trajectoire langagière. Reste que, dans tous les cas, parmi d'autres vecteurs de socialisation, l'école a dans cette affaire un rôle majeur à jouer, ainsi que l'ont montré d'autres contributions à cette journée d'étude.

\section{Vers une éducation plurilingue et interculturelle ? $^{19}$}

\subsection{Approches plurielles}

S'agissant d'en revenir à la question de départ des organisateurs: "Les approches didactiques 'plurielles' se réfèrent-elles à une forme 'universelle' de plurilinguisme ou prennent-elles en compte ces divers 'plurilinguismes'? Comment? Avec quels objectifs ?", tout ce qui précède (et la simple observation de l'état des lieux didactiques et pédagogiques) conduit directement à quelques quasi évidences :

- La diversité des formes de plurilinguismes et la multiplicité des facteurs qui les travaillent excluent une approche didactique uniforme, mais il n'en demeure pas moins que, dans la plupart des contextes et à travers les cultures éducatives, les formes scolaires dominantes prennent rarement en compte cette diversité des plurilinguismes qu'elles accueillent et peinent à intégrer pleinement la dimension plurilingue dans leur projet éducatif (Cavalli, 2007).

- Si on entend par approches didactiques "plurielles", l'éventail de démarches que présentent l'éveil aux langues, l'enseignement bilingue, la didactique intégrée des langues, l'intercompréhension, le travail interculturel (Candelier, 2008), on dispose en effet d'une panoplie de principes et d'outils désormais bien explicités, illustrés et mis à l'épreuve qui tendent tous à favoriser dans l'apprentissage la mise en relation des langues, des formes d'éveil interculturel et le développement d'aptitudes, d'attitudes et de stratégies de portée transversale.

- Cette diversité peut évidemment, en principe, donner lieu à modulations fines de mise en œuvre des approches plurielles selon les contextes et les formes de plurilinguisme que ces contextes présentent; ce qui constitue une forme de réponse à la question didactique posée pour cette journée.

- Mais trois limitations semblent affecter ce potentiel des approches plurielles. Tout d'abord, leurs modalités de combinaison / articulation en vue d'une intégration dans des choix curriculaires contextualisés restent à définir, ces différents courants innovants s'étant développés séparément et ayant des histoires et des champs d'expérimentation différents ${ }^{20}$. Ensuite, force est de constater que ces approches plurielles restent pour l'essentiel à la fois militantes et marginales, rares étant les systèmes éducatifs qui leur reconnaissent une place 
officielle dans leurs programmes ${ }^{21}$. Enfin et surtout, bien que les approches "plurielles" ne laissent pas de côté la langue de scolarisation, cette dernière n'y prend pas la place déterminante et n'y reçoit pas l'attention particulière que les options et analyses qui précèdent (cf. 1.2 et 1.3.) tendent à lui attribuer dans toute configuration des formes de plurilinguismes. pour l'éducation", développé dans le cadre de la division des politiques linguistiques du Conseil de l'Europe et qui a à voir avec nombre des orientations et interrogations que j'ai abordées ici.

50 Je serai d'autant plus bref qu'il a déjà été question de ce projet dans une rencontre antérieure de l'Acedle (Coste, 2008) et que l'essentiel des travaux relatifs à cette entreprise ambitieuse et complexe sont accessibles en ligne et qu'une plateforme dédiée leur sera bientôt affectée (cf. supra, note 10).

\subsection{Langues de l'éducation, langue(s)de scolarisation, plurilinguismes}

$\mathrm{Si}$, pour l'intervention de politique linguistique ou de didactique, on refuse tout à la fois de postuler une forme "universelle" de plurilinguisme et de se satisfaire d'une atomisation en une infinité de plurilinguismes individuels, il est besoin, en particulier dans la perspective de la formation scolaire, d'un ensemble de références partagées permettant de positionner différentiellement les cas particuliers. C'est une des dimensions de ce que propose le projet "Langues de l'éducation / langues pour l'éducation", dont des options majeures peuvent être résumées comme suit ${ }^{22}$ :

- L'éducation plurilingue et interculturelle y est posée comme tout ensemble un projet et un droit, partie intégrante du droit à une éducation de qualité (Cavalli et al., 2009 ; Coste et al., 2009)

- Toutes les langues et variétés présentes dans l'école, qu'elles soient reconnues en quelque manière dans le curriculum ou qu'elles soient simplement partie du répertoire langagier des élèves (et utilisées par exemple dans la cour de récréation), sont considérées comme des "langues de l'éducation" en ce que toutes contribuent ou sont susceptibles de contribuer, que les élèves les possèdent déjà ou les apprennent ou y soient simplement exposés, à leur expérience scolaire de la pluralité linguistique, au développement de leurs capacités plurilingues et à la construction de leurs connaissances et expériences d'apprentissage.

- La (ou les) "langue(s) de scolarisation" opère(nt) comme véhicule ordinaire, partagé et réputé commun, du fonctionnement de l'école; elle(s) y figure(nt) comme matière(s) enseignée(s) en tant que telle(s) et comme medium d'enseignement d'autres matières. Sa/ leur maîtrise importe au premier chef parmi les objectifs de tout projet éducatif.

- L'ouverture à la pluralité des langues et des cultures est tout aussi nécessaire ; et ceci peutêtre moins pour des raisons instrumentales de fonctionnalité pratique que pour des enjeux globaux tenant à l'avenir de nos sociétés.

- Le défi est dès lors de faire en sorte que tout ce qui se pratique en dehors et à côté de la langue de scolarisation bénéficie à cette dernière et, inversement, que la manière dont celleci est travaillée et développée permette aussi l'ouverture sur la pluralité.

- Penser la langue de scolarisation dans la perspective d'une éducation plurilingue consiste ainsi à en "décanoniser" les représentations et les pratiques, tout en soulignant et en 
travaillant les régulations et règles qui en structurent la diversité des usages, et singulièrement en situation scolaire.

- A cette condition, la langue de scolarisation devient la clé de voute de l'éducation plurilingue et interculturelle. Mais complémentairement, cette éducation n'a de chance de se développer dans les systèmes éducatifs que si elle s'y installe aussi au bénéfice de la langue de scolarisation (Perregaux, 2004 ; Bertucci \& Corblin, 2004). entre les établissements quant aux profils linguistiques des élèves accueillis, c'est une gestion locale fine qui semble souhaitable, mais inscrite dans des finalités transversales communes où la pluralité linguistique et culturelle soit non seulement acceptée comme donnée individuelle de départ mais aussi valorisée et développée, tant comme ressource pour la collectivité que comme composante incontournable de toute visée d'inclusion et de cohésion sociales dans nos sociétés contemporaines. Ce qui confirme que la langue de scolarisation, condition forte (parmi d'autres) de la réussite personnelle et vecteur principal de la participation à l'espace public, soit travaillée à la fois "en soi" et comme partie intégrante d'un ensemble de capacités langagières plurielles qu'elle influence et qui l'affectent en retour. situation scolaire à l'intérieur d'un espace social donné, c'est aux relations entre cette langue de scolarisation, les répertoires langagiers des jeunes scolarisés, les autres langues présentes dans l'école et les usages sociaux extérieurs qu'il convient de travailler dans la perspective d'une éducation plurilingue et interculturelle. Selon les contextes et les jeux de critères qui peuvent caractériser les formes de plurilinguismes y ayant cours, les conditions de mise en œuvre de ce rôle de l'école, pour autant qu'il soit reconnu, varient fortement (Cortier, 2006 ; Castellotti, 2008). Mais, par delà cette extrême diversité et quels que soient, si l'on peut dire, les "plurilinguismes d'entrée" et les "plurilinguismes de sortie", il est permis d'estimer que le dispositif d'ensemble, en termes éducatifs et didactiques, présente alors les mêmes enjeux.

terminer, sans conclure, sur un point et un cas particuliers, deux mots des contextes de scolarisation primaire où intervient l'enseignement d'une langue "étrangère". De même que beaucoup se joue au niveau du pré-scolaire, là où il existe, de même l'introduction naguère dite "précoce" d'une langue étrangère joue sans doute un rôle important dans l'avenir de la construction plurilingue, suivant les modalités de sa 
mise en contact avec les autres pratiques langagières de l'école (cf. Les Langues modernes 4/2007).

57 C'est dans ce sens que se situe un des projets de l'ADEB qui travaille, avec des collègues de divers horizons, à concrétiser les principes et propositions formulées dans le document déjà mentionné (Castellotti, Coste et Duverger, 2008) en les ordonnant, pour l'enseignement primaire déjà, autour de trois composantes posées comme complémentaires et interdépendantes entre elles dans une perspective d'éducation plurilingue "globale", où chacune bénéficie aux autres :

- la langue majeure de scolarisation

- la langue "autre" (étrangère, régionale...) enseignée

- les autres dimensions plurilingues et interculturelles (répertoires des élèves et approches plurielles) ${ }^{24}$

L'option retenue est de poser que :

- la langue majeure de scolarisation est une pièce maîtresse de toute éducation plurilingue et interculturelle;

- au moins une langue autre est enseignée "sérieusement" dès le primaire ;

- d'autres éléments d'une éducation plurilingue et interculturelle sont intégrés au curriculum en étroite relation aux deux premiers, mais aussi par le biais des disciplines autres ;

- des rôles distincts existent à l'intérieur de l'ensemble mais aussi de nécessaires circulations et mises en relation;

- pour toutes les composantes, la variabilité linguistique est pleinement prise en considération ;

- le tout s'inscrit dans un processus toujours contextualisé donnant lieu à des formes d'évaluation et d'adaptation. (ADEB, document de travail)

A suivre donc... même si bien des notions restent en question.

Marcellesi, J.-B. (2003). Sociolinguistique : épistémologie, langues régionales, polynomie. Textes réunis par Bulot, T. \& Blanchet, P. Paris : L'Harmattan

\section{BIBLIOGRAPHIE}

Beacco, J.-C. (2005). Langues et répertoire de langues : le plurilinguisme comme "manière d'être" en Europe. Étude de référence, Division des politiques linguistiques. Strasbourg : Conseil de l'Europe. Disponible en ligne. http://www.coe.int.

Beacco, J.-C. \& Byram, M. (2003, édition revisée 2007). Guide pour l'élaboration des politiques linguistiques éducatives en Europe - De la diversité linguistique à l'éducation plurilingue. Strasbourg : Conseil de l'Europe, Division des Politiques linguistiques. Disponible en ligne. http:// www.coe.int/t/dg4/linguistic/Guide_niveau3_FR.asp

Berrendonner, A., Le Guern, M. \& Puech, G. (1983). Principes de grammaire polylectale. Lyon : Presses Universitaires de Lyon.

Bertucci, M.-M. et Corblin, C. (éds.) (2004). Quel français à l'école? Les programmes de français face à la diversité linguistique. Paris : L'Harmattan.

Billiez, J. (2005). "Répertoires et parlers plurilingues. Déplacements à opérer et pistes à parcourir à l'école". In Prudent, L. F., Tupin, F. \& Wharton, S. (éds). Du plurilinguisme à l'école. Vers une gestion 
coordonnée des langues en contextes éducatifs sensibles. Berne - Fribourg : Peter Lang, collection Transversales, pp. 323-340.

Blanchet, P., Calvet, L.-J., De Robillard, D. (2007). Un siècle après le 'Cours' de Saussure. La linguistique en question. Cahiers d'ateliers de sociolinguistique $\mathrm{n}^{\circ} 1$. Paris : L'Harmattan.

Byram, M. (2009). Sociétés multiculturelles et individus pluriculturels : le projet de l'éducation interculturelle. Langues dans l'éducation/langues pour l'éducation. Strasbourg : Conseil de l'Europe, Division des Politiques linguistiques. Accessible en ligne. http://www.coe.int Candelier, M. (dir.) (2003a). Evlang - l'éveil aux langues à l'école primaire. Bruxelles : De Boeck Duculot.

Candelier, M. et al. (2003b). Janua Linguarum - La porte des langues. L'introduction de l'éveil aux langues dans le curriculum. Strasbourg : Éditions du Conseil de l'Europe.

Candelier, M. (2008). "Approches plurielles, didactiques du plurilinguisme : le même et l'autre". Les Cahiers de l'Acedle, volume 5, pp. 65-90.

Castellotti, V. (éd.) (2001). D’une langue à d'autres : pratiques et représentations. Rouen : Presses universitaires de Rouen, DYALANG.

Castellotti, V. (2008). "L'école française et les langues des enfants : quelle mobilisation de parcours plurilingues et pluriculturels ?". In Chiss, J.-L. (Dir.), Immigration, École et didactique du français, Paris : Didier, collection Langues et didactique.

Castellotti, V. \& Moore, D. (2005). "Répertoires pluriels, culture métalinguistique et usages d'appropriation". In Beacco, J.-C., Chiss, J.-L., Cicurel, F. \& Véronique, D. (dir.). Les cultures éducatives et linguistiques dans l'enseignement des langues. Paris : PUF, pp. 107-132.

Castellotti, V., Coste, D., Duverger, J. (éds) (2008). Propositions pour une éducation au plurilinguisme en contexte scolaire. Document ADEB / Dynadiv Université de Tours. Accessible en ligne : http://www.adeb.asso.fr/index_2i.php

Castellotti, V. et al. (2009). "A propos de la notion de compétence plurilingue en relation à quelques concepts sociolinguistiques ou Du rôle de l'implication et de l'intervention dans la construction théorique". In Pierozak, I. \& Eloy, J.-M. (éds). Intervenir : appliquer, s’impliquer? Paris : L'Harmattan, pp. 95-104.

Cavalli, M. (2005). Éducation bilingue et plurilinguisme. Le cas du Val d'Aoste. Paris : Didier, collection LAL.

Cavalli, M. (2007). "Du bilinguisme au plurilinguisme : De nouveaux défis pour les politiques linguistiques éducatives". In Moore, D. et Castellotti, V. (éds). La compétence plurilingue : regards francophones. Berne : Peter Lang, Collection Transversales, pp. 27-50.

Cavalli, M., Coste, D., Crişan A. \& Van de Ven, P. H. (2009). L'éducation plurilingue et interculturelle comme projet. Langues dans l'éducation/langues pour l'éducation. Strasbourg : Conseil de l'Europe, Division des Politiques linguistiques. Accessible en ligne. http://www.coe.int/t/dg4/ linguistic/Source/LE_texts_Source/EducPlurInter-Projet_fr.pdf

Cenoz, J. \& Genesee, F. (eds) (1998). Beyond Bilingualism, Multilingualism and Multilingual Education. Clevedon : Multilingual Matters.

Conseil de l'Europe (2001). Cadre européen commun de référence pour les langues : Apprendre, enseigner, évaluer. Paris : Didier. Disponible en ligne. http://www.coe.int/fr/web/commoneuropean-framework-reference-languages 
Cortier, C. (2006). "FLS à l'école : d'un bilinguisme de transition à la promotion du plurilinguisme". Éducation et sociétés plurilingues, $\mathrm{n}^{\circ}$ 19, pp. 31-52.

Coste, D. (2004). "De quelques déplacements opérés en didactique des langues par la notion de compétence plurilingue". In A. Auchlin et al. (éds). Structures et discours. Mélanges offerts à Eddy Roulet. Québec : Nota Bene. pp. 67-85.

Coste, D. (2008). "Éducation plurilingue et langue de scolarisation". Les Cahiers de l'Acedle, volume 5, pp. 91-107.

Coste, D. (2009). "Médiation et altérité". LIDIL nº 39, pp. 163-170.

Coste, D., Moore, D. \& Zarate, G. (1997). Compétence plurilingue et pluriculturelle. Vers un Cadre Européen Commun de référence pour l'enseignement et l'apprentissage des langues vivantes. Études préparatoires. Strasbourg: Conseil de l'Europe.

Coste, D., Cavalli, M., Crişan, A. \& Van de Ven, P.H. (2009). L'éducation plurilingue et interculturelle comme droit. Langues dans l'éducation/langues pour l'éducation. Strasbourg: Conseil de l'Europe, Division des Politiques linguistiques Accessible en ligne. www.coe.int/t/dg4/linguistic/Source/ LE_texts_Source/EducPlurInter-Droit_fr.pdf

Dabène, L. (1994). Repères sociolinguistiques pour l'enseignement des langues. Paris : Hachette (collection F).

Lüdi, G. (2004). "Pour une linguistique de la compétence du locuteur plurilingue". Revue française de linguistique appliquée, IX-2, pp. 125-135.

Lüdi, G. \& Py, B. (2002). Etre bilingue. Berne : Peter Lang.

Les Langues modernes 4/2007. "Dossier : Les langues en Primaire : quelles articulations"

Moore, D. (2006). Plurilinguismes et école. Paris : Didier, Collection LAL.

Moore, D. \& Castellotti, V. (2008). "Perspectives de la recherche francophone autour de la notion de compétence plurilingue". In La compétence plurilingue : regards francophones. Berne - Fribourg : Peter Lang, collection Transversales, pp. 11-24.

Perregaux, C. (2004). "Prendre appui sur la diversité linguistique et culturelle pour développer aussi la langue commune". Repères $\mathrm{n}^{\circ} 29, \mathrm{pp}$. 147-166.

Prudent, L. F. (2005). "Interlecte et pédagogie de la variation en pays créoles". In Prudent, L. F., Tupin, F. \& Wharton, S. (éds). Du plurilinguisme à l'école. Vers une gestion coordonnée des langues en contextes éducatifs sensibles. Berne - Fribourg : Peter Lang, collection Transversales, pp. 359-378.

Prudent, L. F., Tupin, F. \& Wharton, S. (éds) (2005). Du plurilinguisme à l'école. Vers une gestion coordonnée des langues en contextes éducatifs sensibles. Berne - Fribourg : Peter Lang, collection Transversales.

Renaud, P. (1998)."Absoute pour un locuteur natif". Le français en Afrique ${ }^{\circ} 12$. Disponible en ligne : http://www.unice.fr/ILF-CNRS/ofcaf/12/12.html

Stratilaki, S. (2008). "Composantes, structure opératoire et dynamique de la compétence plurilingue ; modes d'articulation et formes de construction". In Moore, D. \& Castellotti, V. (dir.). La compétence plurilingue : regards francophones. Berne - Fribourg: Peter Lang, collection Transversales, pp. 51-82.

Truchot, Cl. (2008). Europe ; l'enjeu linguistique. Paris : La documentation française. 
Vollmer, H. (2006a). Vers un instrument européen commun pour la/les langue(s) de scolarisation. Langues dans l'éducation/langues pour l'éducation. Strasbourg: Conseil de l'Europe, Division des Politiques linguistiques Accessible en ligne. https://rm.coe.int/16805c7463

Vollmer, H. (2006b). Langues d'enseignement des disciplines scolaires. Langues dans l'éducation/ langues pour l'éducation. Strasbourg : Conseil de l'Europe, Division des Politiques linguistiques Accessible en ligne. https://www.coe.int/t/dg4/linguistic/Source/Vollmer_LAC_FR.doc

Zarate, G., Lévy, D., Kramsch, Cl. (dir.) (2008). Précis du plurilinguisme et du pluriculturalisme. Paris : Éditions des archives contemporaines.

\section{NOTES}

1. Pour une vue d'ensemble à la fois informative et problématisée, voir Europe : l'enjeu linguistique (Truchot, 2008)

2. L'individu plurilingue n'ayant rien du polyglotte émérite, mais se caractérisant - dans les définitions les plus communes - par la maîtrise plus ou moins fonctionnelle, à des degrés très divers, de plusieurs langues (cf. Cadre européen commun de référence pour les langues, p. 129.

3. A cet égard, à un moment où l'accent a été résolument mis par le Commissariat au multilinguisme sur la traduction, il est permis de se demander si le message n'est pas aussi que la combinaison "langue première + langue étrangère internationale + traduction d'autres langues vers la langue première ou vers la langue étrangère internationale" est, nonobstant les déclarations de principe plus ambitieuses, considérée, la plus réaliste, voire la plus souhaitable pour les citoyens européens. Et, à pousser un peu plus loin (trop loin ?) cette interprétation, on trouve les déclarations, récurrentes dans des discours de responsables politiques de haut niveau (par exemple, naguère, en Italie ou en France), selon lesquelles l'école a pour finalité de produire de "bons" bilingues; l'objectif officiel et toujours affiché de l'Union européenne ("langue première + deux autres langues") risquant alors de passer quelque peu au second plan des priorités.

4. Au point, ici ou ailleurs, de recommander aux mères, si elles tiennent à la réussite scolaire de leurs enfants, de ne pas pratiquer en famille la langue maternelle.

5. L'ouverture de l'Europe depuis 1989, l'attention portée aux droits linguistiques des minorités, les affirmations des langues régionales ont contribué à "conscientiser" ce plurilinguisme ordinaire. D'un bord, ce plurilinguisme de fait a pu être valorisé comme une ressource et une chance à ne pas négliger, tant pour les personnes que pour les divers pays et pour l'Europe entière. Cependant que, d'un autre côté, l'accroissement du nombre des pays membres de l'Union européenne et donc des langues officiellement reconnues (sans même parler des autres) par l'Union apparaissait comme une cause entre autres de complexification et d'alourdissement de la gestion institutionnelle.

6. A cet égard, la distinction conceptuelle établie par L. Dabène (1994) entre parler vernaculaire, langue de référence, langue d'appartenance garde toute sa pertinence.

7. La communication est exolingue lorsque les divergences entre les répertoires linguistiques respectifs des interlocuteurs apparaissent comme constitutives du fonctionnement de l'interaction, c'est-à-dire lorsque le recours à des procédés d'ajustement réciproque, d'auto/ hétérofacilitation, etc., devient un trait saillant de la communication. Elle devient endolingue, au contraire, lorsque les divergences codiques ne représentent plus une donnée pertinente dans la gestion du discours, autrement dit lorsqu'elles ne sont plus perçues comme significatives par les participants à l'événement langagier. Les initiateurs de ces notions les posent comme désignant des pôles entre lesquels la communication peut se positionner de manière variable (y compris au cours d'une même conversation). 
8. De divers bords, la notion de langue est interrogée et mise en question(s). On y voit un artefact historiquement établi qui ne peut rendre pleinement raison de la variabilité des formes et de la multiplicité des pratiques langagières, seules empiriquement attestées. Mais, quels que soient les enjeux théoriques et autres de ces interrogations, on ne se débarrasse ni facilement ni impunément d'un objet social aussi "naturalisé" et à aussi forte charge symbolique dans les représentations et les discours. Autant il est nécessaire d'insister sur la variation et les variétés, sur le caractère pluriel, de toute "langue", autant, dans une perspective d'enseignement et d'éducation, y compris plurilingue, la notion de langue semble, certes insuffisante et inadéquate, mais aussi incontournable.

9. Caractérisation quelque peu métaphorique qui n'est pas sans analogie avec celles appliquées naguère à l'interlangue, lorsque cette dernière était vue comme composée de sous-systèmes interreliés ou non d'accessiblité, de stabilité, de cohésion et de cohérence internes variables, n'évoluant pas au même rythme ni d'un même mouvement dans leur accommodation des données de la langue "cible". Mais cette analogie, outre qu'elle s'effectue en effet sur ce qui était qualifié plus haut de terrain mouvant, permet aussi de mettre en évidence des différences notables par delà une certaine similarité des métaphores: d'une part, s'agissant de la représentation de le compétence plurilingue et des ressources auxquelles elle fait appel, l'horizon n'est pas (seulement) l'acquisition d'une langue seconde et, en tout état de cause, cette dernière est elle-même conçue comme présentant une hétérogénéité et une variabilité analogues à celles attribuées à l'interlangue ; d'autre part, on ne considère pas (seulement) une phase en quelque sorte transitoire d'acquisition mais le fonctionnement ordinaire des échanges interactionnels; enfin, la complexification de la notion de compétence plurilingue porte - on vient de le noter - sur des dimensions autres aussi que linguistiques.

10. Comme tous les documents résultant des activités de la Division des politiques linguistiques du Conseil de l'Europe, les "Profils" établis sont accessibles en ligne (http://www.coe.int/T/DG4/ Linguistic/Default_fr.asp)

11. Avec des questions portant sur la diversification des langues proposées par le système scolaire, la continuité / discontinuité entre les cycles d'études, la mise en œuvre des niveaux de référence du Cadre européen commun de référence, l'organisation du curriculum, etc.

12. En Norvège, deux variétés du norvégien (bokmål et nynorsk) et présence territoriale de variétés du sámi ; autres langues de scolarisation pour certaines minorités en Lituanie (polonais et russe), en Estonie (essentiellement russe), en Slovaquie (essentiellement, mais pas seulement, hongrois); langue officielle nationale (gaëlique) distincte de la langue majeure de scolarisation (anglais) en Irlande.

13. Toutes ces autres langues ont été des langues de la domination et ont, à des degrés divers, minorisé la langue aujourd'hui nationale et de scolarisation majeure; s'agissant de la Slovaquie, on doit aussi tenir compte du voisinage du tchèque et de la volonté, de part et d'autre, de souligner des spécificités que l'on tendait à moins mettre en avant du temps de l'entité hybride tchéco-slovaque.

14. Pour ne prendre qu'un exemple, il suffit de noter que, dans bon nombre de pays européens aujourd'hui, l'accueil scolaire d'enfant d'immigrants pour lesquels la langue de scolarisation est seconde affecte en profondeur ou à tout le moins interroge les pratiques d'enseignement de la langue de scolarisation.

15. Mais à l'inverse, si l'on peut dire, il n'est pas rare que les méthodes actives et parfois ludiques utilisées pour les langues étrangères (et tout particulièrement pour l'anglais) soient comparées par les élèves avec les pratiques plus normatives, perçues comme formelles ou puristes, de l'enseignement de la langue de scolarisation comme matière et entrainent une baisse de motivation, voire une désaffection pour l'apprentissage de cette dernière. 
16. De manière peut-être plus étonnante, même l'anglais, partout désigné comme langue à part dans son statut de première langue étrangère "écrasant" les autres, ne provoque ni le même type d'attraction ni les mêmes inquiétudes en Norvège qu'en Lituanie.

17. Pour une étude de cas soulevant nombre de questions transversales, voir Cavalli 2005.

18. Pour autant, il se peut que la compétence plurilingue des slovaquophones majoritaires soit d'une certaine manière plus étendue, du fait des degrés d'intercompréhension qui existent avec non seulement le tchèque (bien évidemment) et le russe (pour certaines générations), mais aussi avec le polonais et l'ukrainien. Le paradoxe est que ces proximités avec des langues à plus d'un titre "voisines" ne sont ni valorisées ni explicitement cultivées dans la formation scolaire. Peutêtre ont-elles toutefois une certaine influence sur les représentations et pratiques du slovaque pour les "natifs" : bien que l'enseignement du slovaque, longtemps langue dominée, soit assez normatif, il ne semble donner lieu ni aux mêmes prescriptions formelles du législateur ni aux mêmes insécurités induites chez les locuteurs que le lituanien en Lituanie ou l'estonien en Estonie, langues plus "singulières". Mais d'autres facteurs sont aussi à l'œuvre...

19. La dimension interculturelle sera minorée dans ce qui suit. Non de propos délibéré, mais en raison de l'objet de cette contribution, centrée sur les plurilinguismes. Suffit peut-être de noter ici que, dans les travaux relatifs à l'éducation plurilingue et interculturelle, cette dimension est bien présente et de plus en plus articulée aux pratiques langagières. Voir notamment à cet égard Byram 2009. En dehors des travaux du Conseil de l'Europe, on trouve aussi une forte insistance sur les aspects (inter)culturels dans le Précis du plurilinguisme et du pluriculturalisme (Zarate, Lévy \& Kramsch, 2008). On rappellera que, dans le CECR, il était question seulement d'une "compétence plurilingue et pluriculturelle". L'éducation plurilingue et interculturelle en vient aussi à poser que tout acteur social est de fait un plurilingue en devenir et participe de plusieurs cultures, mais le passage de "pluriculturel" à "interculturel" implique un autre niveau de compréhension, de réflexivité, de mise en relation et en perspective des phénomènes et des comportements culturels dans leur diversité. C'est aussi le développement de compétences de médiation, notamment langagière, des différences, des chocs et des conflits entre cultures. Ajoutons que, rétrospectivement, il est permis de considérer que, au moment de la publication du CECR, l'affirmation du caractère pluriculturel de l'acteur social était sans doute plus novatrice et plus radicale que l'insistance sur une interculturalité qui avait déjà donné lieu à focalisations multiples dans le champ éducatif.

20. On doit cependant souligner que, dans ces développements successifs et dans certaines de ces réalisations, l'éveil aux langues s'avère plus intégratif, notamment quant aux formes de travail sur l'intercompréhension entre langues voisines, la didactique intégrée des langues et les dimensions interculturelles, la mise en perspective curriculaire d'ensemble restant le point sensible (Candelier, 2003a, 2003b).

21. C'est sans doute l'enseignement bilingue qui a le plus connu une reconnaissance large dans des contextes divers marqués par la présence de langues minoritaires ou régionales, des affirmations "autonomes" locales (Catalogne, Pays basque, Val d'Aoste, etc.) ou des choix de plusieurs langues de scolarisation au niveau national (Luxembourg). Dans les cas plus "ordinaires", les extensions notables sont aussi celles de formes d'enseignement bilingue, qui, en Europe, bénéficient surtout à l'anglais, avec le CLIL (Content and Language Integrated Learning) et, dans une moindre mesure et grâce à des soutiens officiels volontaristes, au français, avec l'EMILE (Enseignement de Matières par Intégration d'une Langue Etrangère). Mais il est facile d'observer, d'un côté, que c'est justement ce rapport à l'enseignement d'une langue et à son intégration aux autres matières scolaires que l'éveil aux langues / ouverture aux cultures, pour de large portée qu'il soit, n'inclut pas dans son champ de possibles et, d'un autre côté, qu'une forme d'enseignement bilingue telle que le CLIL doit surtout son succès à des représentations sociales et des visées éducatives tenant plus du bilinguisme (aussi "parfait" que possible) que d'une prise en compte et d'un développement des capacités plurilingues des apprenants (cf. 
aussi, supra, note 3). Complémentairement, on relèvera que le projet fort d'éducation langagière (Educazione linguistica) naguère développé en Italie a fait long feu et que, toujours en Italie, des orientations officielles (Indicazioni) qui mettent en relation variétés de l'italien, langues de l'immigration, langues étrangères ne donnent lieu qu'à des expérimentations d'avenir incertain, cependant que sont entrés dans les faits l'anglais précoce obligatoire pour tout le monde et la possibilité de l'emploi des heures de la deuxième langue communautaire pour de l'anglais renforcé.

22. Les points qui suivent empruntent des formulations tant à des documents du projet "Langues de l'éducation" (http://www.coe.int/T/DG4/Linguistic/Default_fr.asp) qu'à des développements figurant dans le texte collectif résultant d'un séminaire organisé par l'ADEB (Association pour le développement de l'éducation bi-plurilingue) et l'équipe Dynadiv de l'Université de Tours (Castellotti, Coste \& Duverger, 2008). On se reportera aussi, ainsi que mentionné ci-dessus, à la communication publiée dans le $\mathrm{n}^{\circ} 5$ des Cahiers de l'Acedle (Coste, 2008).

23. De même et plus encore que pour la dimension interculturelle (cf. note 19), il y a lieu de bien marquer que l'orientation de la présente contribution fait que la place des autres matières que les langues dans l'éducation plurilingue et interculturelle s'y trouve indûment minorée. Ces "autres" matières ont toute leur place dans le projet éducatif. Ce d'autant plus que, comme le montre Vollmer (2006a, 2006b), outre les apports de leurs contenus spécifiques, elles contribuent à l'extension du répertoire langagier des apprenants et les introduisent tant soit peu à des cultures de communautés de pratiques particulières. A bien des égards, le développement plurilinguistique et pluriculturel en contexte scolaire passe aussi fortement par le travail de ces disciplines.

24. Cette structuration simple présente l'avantage (y compris pour ce qui est des développements au-delà du primaire) de tenir compte plus explicitement des matières du programme, voire des spécialités des enseignants.

\section{RÉSUMÉS}

Cette contribution aborde la pluralité des plurilinguismes individuels et propose quelques critères de nature à catégoriser cette pluralité. Elle présente ensuite un retour sur l'évolution de la notion de compétence plurilingue et commente quelques aspects d'analyses de politique linguistique de différents pays européens. Elle souligne enfin les perspectives ouvertes par une éducation plurilingue et interculturelle qui tout à la fois prendrait la pleine mesure de cette pluralité des plurilinguismes et se donnerait des repères et des principes d'action autres que purement contextuels.

This paper addresses the plurality of individual plurilingualism by first suggesting a few categorizing criteria for this diversity. It then retraces some changes the notion of plurilingual competence has undergone and comments on the results of analyses of the language policies of several European countries. Finally it underlines the potential for a plurilingual and intercultural education which would take this plurality of plurilingualism into full consideration, while calling on principles and guidelines other than those that are merely context bound. 
INDEX

Mots-clés : plurilinguisme, compétence, éducation, Europe

Keywords : plurilingualism, competence, education, Europe

\section{AUTEUR}

\section{DANIEL COSTE}

Daniel Coste est professeur émérite à l'École normale supérieure Lettres et sciences humaines, membre de l'équipe ICAR, et directeur de recherches à l'Université Paris 3 - Sorbonne nouvelle, membre de l'équipe DILTEC. Ancien directeur du CREDIF (Centre de Recherche et d'Étude pour la Diffusion du Français) et responsable de l'équipe de recherche "Plurilinguisme et apprentissages". A été professeur ordinaire de linguistique appliquée à l'Université de Genève et directeur de l'école de langue et de civilisation françaises de cette même université. Est actuellement associé à certains des travaux de la Division des politiques linguistiques du Conseil de l'Europe.

Courriel : dcoste[at]ens-lsh.fr ; dlcoste2[at]wanadoo.fr

Adresse : ENS-LSH, parvis René Descartes, BP 7000, 69342 Lyon cedex 07 